\title{
Caractérisation micro-echelle du risque d'inondation : modélisation hydraulique détaillée et quantification des impacts socio-économiques
}

\author{
Benjamin J. DEWALS ${ }^{1}$, Sylvain DETREMBLEUR, Pierre ARCHAMBEAU, Sébastien ERPICUM, \\ Julien ERNST, Michel PIROTTON
}

\author{
Université de Liège, Unité d'Hydrologie, Hydrodynamique Appliquée et Constructions Hydrauliques - HACH \\ 1. Fonds de la Recherche Scientifique F.R.S-FNRS \\ Chemin des Chevreuils, 1 -Bât.B52/3 -B-4000Liège,Belgique,,e-mail : b.dewals@ulg.ac.be, hach@ulg.ac.be
}

\begin{abstract}
RÉSUMÉ. - La communication expose une approche cohérente pour l'analyse du risque d'inondation à micro-échelle. La méthodologie décrite s'appuie sur la cartographie détaillée de l'exposition réalisée par un modèle hydrodynamique 2D ainsi que sur des données d'occupation du sol à résolution géométrique et qualité sémantique élevées. La modélisation de l'écoulement est basée sur les équations en eaux peu profondes, résolues par le biais d'un schéma volumes finis sur maillages multiblocs structurés. Grâce à l'utilisation de données altimétriques laser de haute précision, les simulations sont à même de représenter la dynamique de l'écoulement sur une topographie complexe telle qu'un environnement urbain.

La première partie de la rédaction est consacrée à la description de la méthodologie d'évaluation du risque en commençant par la modélisation de l'aléa et ensuite l'évaluation de la vulnérabilité des biens et des personnes. L'échelle d'analyse de la procédure permet son développement en suivant la philosophie orientée objet : chaque élément soumis au risque étant ainsi considéré comme un objet distinct avec des propriétés et des caractéristiques définies.

Dans un second temps, l'applicabilité de la procédure est démontrée au moyen d'une étude de cas pour laquelle trois mesures de protection contre les inondations sont évaluées. L'analyse détaillée de ces mesures met en exergue des influences sensiblement différentes sur les courbes du risque d'inondation.
\end{abstract}

Mots-clés : inondation, risque inondation, modélisation, analyse du risque, cartographie, vulnérabilité.

\section{Micro-scale characterization of flood risk : detailed inundation modelling and evaluation of socio-economic impacts}

\begin{abstract}
The paper presents a consistent micro-scale flood risk analysis procedure, relying on detailed 2D inundation modelling as well as on high resolution topographic and land use database. The flow model is based on the shallowwater equations, solved by means of a finite volume scheme on multiblock structured grids. Using highly accurate laser altimetry, the simulations are performed with a typical grid spacing of $2 \mathrm{~m}$, which is fine enough to represent the flow at the scale of individual buildings.

The methodology is first described, both in terms of hydraulic modelling and evaluation of people and assets vulnerability. The analysis scale of the procedure enables its development following an object-oriented approach. Finally, the applicability of the procedure is demonstrated through a case study for which three different protection measures are evaluated. Detailed analysis of these measures highlights significantly different influences from those measures on the risk curves.
\end{abstract}

Key-words : flood, flood risk, modelling, risk analysis, topography, vulnerability.

\section{INTRODUCTION}

L'adaptation des populations riveraines des cours d'eau face au risque d'inondation est en perpétuelle évolution. Ce risque qui, au départ était accepté et subi par tous, doit de nos jours être de plus en plus contrôlé et quantifié. Le but de la démarche présentée dans cet article est de minimiser les impacts des crues dans des zones où la pression urbanistique est de plus en plus forte.

Le moyen privilégié de lutte contre les inondations est actuellement la mesure de protection. Qu'elle soit structurelle ou non-structurelle, curative ou préventive, son dimensionnement est lui aussi en pleine évolution. Avec les récents développements des systèmes d'information géographique et des capacités de calcul, l'approche de dimensionnement his- toriquement utilisée pour les ouvrages de protection contre les inondations (empiriques ou basées sur une seule période de retour) est actuellement remplacée par des méthodologies visant à quantifier le risque d'inondation. De plus, dans l'optique de l'évolution du climat, de nombreux modèles convergent non seulement vers une augmentation de la valeur des pics de crue, mais également de la fréquence d'occurrence de ceux-ci.

C'est dans ce cadre très actuel que se situe la présente communication. En effet, elle décrit une filière innovante d'évaluation du risque d'inondation à micro-échelle associé à une modélisation bidimensionnelle sur maillage très fin (typiquement $2 \mathrm{~m}$ ) de l'inondation sur l'ensemble de la plaine inondée. Un tel niveau de détail dans la cartographie des paramètres de l'inondation permet d'exploiter la grande 
qualité sémantique et géométrique des bases de données géographiques actuelles (ex. occupation du sol). Pour ce faire, un modèle hydrodynamique bidimensionnel permet de représenter fidèlement la dynamique de l'écoulement sur une topographie complexe telle qu'un réseau de rues et d'autres éléments structuraux en sur sol.

L'article s'articule en deux parties. La première est consacrée à la description des deux principales composantes d'une analyse de risque. D'une part, la méthode de modélisation de l'aléa inondation est présentée (combinaison de la cartographie de l'inondation et de la probabilité d'occurrence associée à cette crue). D'autre part, la procédure de quantification de la vulnérabilité socio-économique des biens et personnes est également décrite.

La seconde partie décrit l'application de la méthodologie à un cas d'étude localisé sur l'Ourthe, un des principaux affluents de la Meuse. La procédure utilisée pour déterminer la récurrence statistique des crues ainsi que le modèle hydrodynamique (WOLF2D) sont tout d'abord brièvement dépeints. Ensuite, les différentes composantes de l'analyse de l'exposition (combinaison de la cartographie de l'inondation et des bases de données permettant de localiser les biens et personnes soumises au risque d'inondation) et du modèle de vulnérabilité socio-économique appliqué au cas d'étude sont décrites. Enfin, la conclusion expose les particularités novatrices de cet outil d'évaluation du risque d'inondation ainsi que les principales voies d'évolution de la méthodologie.

\section{MODÉLISATION DE L'ALÉA}

\section{II.1. Détermination de la statistique de crue}

L'aléa associé à un phénomène naturel est défini comme la combinaison d'une intensité de danger et d'une probabilité de dépassement. Cette définition peut aisément être particularisée à l'aléa inondation : le premier terme est décrit par les caractéristiques hydrauliques de l'inondation (hauteur d'eau, vitesse, durée de la crue, vitesse de montée des eaux, ...), tandis que la probabilité de dépassement de la crue est typiquement exprimée sous la forme d'une période de retour en années. Celle-ci peut être dérivée d'un traitement statistique des séries de données d'observations ou encore d'une modélisation hydrologique continue du bassin versant, au départ de séries temporelles de pluie.

Dans le cadre d'une analyse de risque, il est admis que l'étude d'un seul événement n'est pas suffisante pour représenter le risque. En effet, comme il sera démontré dans la suite, plusieurs débits de crue adéquatement sélectionnés doivent être modélisées afin de représenter l'ensemble de la courbe de risque [1].

\section{II.2. Modélisation hydrodynamique de la crue}

Il existe un grand nombre d'approches différentes pour la modélisation des évènements de crue en rivière. Depuis divers modèles simplifiés, jusqu'aux modèles hydrauliques unidimensionnels [2] et bidimensionnels, le choix d'une approche de modélisation doit être cohérente avec l'échelle de l'analyse de risque sous jacente [3]. Dans le cadre d'une approche micro-échelle, la représentation de la dynamique de l'écoulement revêt une importance capitale dans l'optique d'une quantification du risque pour laquelle chaque élément soumis à l'aléa est analysé individuellement. A fortiori, dans un environnement urbain où les hauteurs d'eau et le champ de vitesses sont très hétérogènes, la combinaison d'un modèle $2 \mathrm{D}$ et d'une donnée altimétrique à très haute résolution s'avère plus indiquée. De plus il a été démontré que dans le cas d'une analyse atteignant un tel niveau de détail, les interactions hydrodynamiques entre le lit majeur et le lit mineur ne peuvent être négligées [4] comme ce le serait avec un modèle unidimensionnel ou un modèle simplifié.

\section{MODÉLISATION DE LA VULNÉRABILITÉ}

\section{III.1. Exposition}

L'exposition consiste à identifier les éléments soumis au risque en combinant adéquatement les bases de données identifiant les enjeux étudiés et la cartographie des caractéristiques de l'inondation. Dans le cadre d'une méthodologie micro-échelle, chaque élément soumis au risque peut être considéré comme un objet. Il est localisé par la base de données d'occupation du sol et des propriétés lui sont assignées, parmi lesquelles la valeur estimée de l'objet, son adresse postale, son type, le nombre de personnes qui y habitent s'il s'agit d'une habitation, ...

L'assignation de ces paramètres sur base des résultats d'un modèle hydraulique $2 \mathrm{D}$ détaillé présente une difficulté particulière puisque que le modèle numérique de surface utilisé comme base à la simulation hydraulique inclut les obstacles hors sol qui influencent l'écoulement. En conséquence, les paramètres hydrauliques à assigner à un bâtiment ne peuvent pas être déterminés de manière univoque par superposition de la cartographie de l'inondation et des bases de données. Ils doivent dès lors être déterminés par une analyse de voisinage de l'objet avant de lui être assignés en tant que propriétés [5], tel que le détaille la section IV.6.

\section{III.2. Impacts sociaux}

Trois facteurs majeurs sont à considérer afin de quantifier la vulnérabilité sociale: les paramètres de l'inondation $(F I)$, la vulnérabilité des personnes soumises au risque $(V)$ et la capacité d'adaptation des communautés $(A C)$ [6-8]. Ces trois facteurs sont ensuite combinés de la manière suivante afin d'évaluer l'index d'impact social $(S I)$, $S I=0.5 F I+0.25 V+0.25 A C$. L'impact social d'une inondation peut dès lors être exprimé par le nombre de personnes touchées selon trois catégories d'impact : faible $(0<\mathrm{SI} \leq 0.33)$, moyen $(0.33<\mathrm{SI} \leq 0.66)$, et élevé $(0.66<\mathrm{SI})$.

Les différents indices sont calculés comme suit. Tout d'abord, la hauteur d'eau, la vitesse, la vitesse de montée des eaux et la durée de l'inondation sont combinées afin de calculer un indice de crue. Ensuite, les indicateurs de la vulnérabilité, au nombre de six, sont calculés à partir du ratio du nombre de personnes âgées, de personnes en mauvaise santé, de familles monoparentales, d'étrangers, de personnes défavorisées et de personnes vivant dans des habitations à un seul étage parmi la population totale. Ces indicateurs sont agrégés, par une moyenne géométrique et des facteurs pondérateurs identiques, en un indice unique. Contrairement à la vulnérabilité sociale, la quantification de la capacité d'adaptation est assez récente et peu de recherches s'attachent à la décrire. Toutefois, une méthodologie analytique de détermination de celle-ci à l'échelle des communes de Belgique a été mise au point par Coninx [6]. L'analyse est basée sur plusieurs facteurs, dont l'existence ou non d'une communication organisée sur le risque ou encore l'exis- 
tence de mesures techniques ou non-techniques de protection domestique.

\section{III.3. Dommage économique}

Une approche standard d'évaluation des dommages économiques consiste en l'utilisation de fonctions de dommage [9]. Celles-ci établissent le lien entre le dommage induit à un type de bien particulier par la crue et les paramètres hydrauliques, en particulier les hauteurs d'eau et les vitesses [10-12]. Bien que d'autres paramètres influencent les dommages tels que les mesures de précaution, la durée de la crue ou encore la contamination de l'eau par des polluants [13]. Seules les hauteurs d'eau et les vitesses sont généralement prises en compte dans ces fonctions.

Dans le cadre d'une analyse micro-échelle l'utilisation de fonctions de dommage relatif (dommage exprimé comme une fraction de la valeur du bien) permet, contrairement aux fonctions absolues, de tenir compte de la variabilité locale de la valeur des biens par l'intermédiaire de l'estimation de celle-ci.

L'objectif de cette recherche n'étant pas de développer un nouveau modèle de dommages, nous avons utilisé le modèle récent FLEMO - Flood Loss Estimation Model développé par

Kreibich et al. [14] et Thieken et al. [15], ainsi que les dernières évolutions de celui-ci FLEMOps - Flood Loss Estimation Model for the private sector. Ce modèle est valide pour une approche micro-échelle [3].

\section{CAS D'ÉTUDE}

\section{IV.1. Présentation}

Avec un bassin versant de $2900 \mathrm{~km}^{2}$, l'Ourthe est le principal affluent de la Meuse en Belgique. Les tronçons étudiés sont localisés quelques kilomètres en amont de la confluence avec la Meuse, située à hauteur de Liège en Région wallonne. Trois mesures de protection contre les inondations par débordement de cours d'eau ont été analysées afin d'atténuer les impacts des inondations subies par trois villages (Tilff, Esneux et Poulseur). Les mesures prises en compte ont été choisies à la suite d'une étude de la dynamique des crues et en concertation avec le gestionnaire du cours d'eau. À Tilff, la mesure de protection consisterait à réhabiliter un canal anciennement exploité pour la navigation fluviale et ainsi augmenter la section du cours d'eau. La deuxième mesure, envisagée à Esneux, vise à installer des rehausses mobiles au dessus d'un mur de protection existant, initialement dimensionné pour protéger le village contre une crue de récurrence 20 ans. Enfin, les modélisations hydrauliques de débits de crue à Poulseur ont révélé la présence d'une plaine inondée passive, où les vitesses sont très faibles voire nulles. La mesure consisterait dès lors à modifier la configuration de la topographie à l'entrée et la sortie de cette zone, afin de permettre aux vitesses de se développer et ainsi d'augmenter la section de passage effective du cours d'eau.

\section{IV.2. Détermination de la statistique des débits modélisés}

L'Ourthe étant assez bien instrumentée, il a été possible d'exploiter une série de 30 années d'observations horaire à une station limnimétrique localisée juste à l'aval du secteur étudié. À partir des maximas annuels de débits horaires, une distribution statistique de type gamma a été ajustée. Les deux paramètres ont été calés par maximum de vraisemblance (le paramètre d'échelle vaut $0,01464\left(\mathrm{~m}^{3} / \mathrm{s}\right)^{-1}$ et le paramètre de forme 5,809). Etant donné la configuration des différents tronçons étudiés, aucun apport en long significatif n'est considéré. La loi liant le débit en fonction de la probabilité de dépassement est donc identique pour les trois tronçons étudiés.

\section{IV.3. Modélisation hydrodynamique de la crue}

Tel que démontré par de Wit et al. [16], les affluents de la Meuse dans les Ardennes belges sont caractérisés par d'importants gradients topographiques. Ceux-ci, combinés à des vallées assez étroites, induisent une très faible capacité de stockage dans les plaines inondables de ces rivières. Si on considère un évènement de crue typique sur l'Ourthe, nous avons vérifié que le volume d'eau stocké dans les plaines inondables le long d'un tronçon d'une dizaine de kilomètres reste inférieur à un pourcent du volume total débordant pendant la crue. De plus, la comparaison des emprises maximales d'inondation simulées en suivant ou non l'hypothèse d'un débit stationnaire amène à la conclusion que les deux approches convergent vers des résultats identiques. L'ensemble de ces éléments justifie donc le choix d'une approche stationnaire pour la modélisation de débits de crue sur les tronçons de l'Ourthe. En outre, l'hypothèse de stationnarité de la crue permet de réduire de manière substantielle le temps de calcul nécessaire à la modélisation grâce à des procédures améliorant la vitesse de convergence tel que le raffinement séquentiel du maillage.

Le modèle hydraulique bidimensionnel WOLF2D a été exploité pour cette étude. Entièrement développé au sein de l'unité de recherches du HACH à l'Université de Liège, il a été validé et exploité sur la modélisation de plus de $1000 \mathrm{~km}$ de rivière en Belgique. WOLF2D résout les équations en eaux peu profondes par un schéma volumes finis [17-21]. La combinaison du modèle bidimensionnel avec une topographie LIDAR à une résolution de 2 mètres en fait un outil idéal pour modéliser des crues dans les environnements urbains. Les Figure 1 et Figure 3 rendent compte de l'intérêt et de la qualité des résultats de la modélisation numérique. La modélisation de l'aléa apporte également une information de premier ordre pour l'analyse de l'influence des différentes mesures de protection sur la dynamique de l'écoulement. En particulier, dans le cas de la rehausse du mur (Figure 1), le différentiel de hauteur d'eau met en avant une mise en charge à l'amont du mur pour les débits importants (zone colorée en rouge). De même pour la réhabilitation de l'ancien canal, la Figure 2 montre qu'un mur de protection existant à l'aval de Tilff, initialement dimensionné pour un débit centennal deviendrait, grâce au canal, effectif jusqu'à une période de retour légèrement supérieure à 200 ans.

\section{IV.4. Exposition}

Deux bases de données complémentaires ont été exploitées pour identifier les enjeux exposés. D'une part, la base de données du PICC (Plan Intercommunale de Cartographie Continue) est caractérisée par une très haute résolution géométrique et une qualité sémantique raisonnable. Afin de palier à cette limitation, la base de données du Cadastre permet de compléter l'information sémantique contenue dans le PICC selon une procédure automatisée par jointure spatiale 

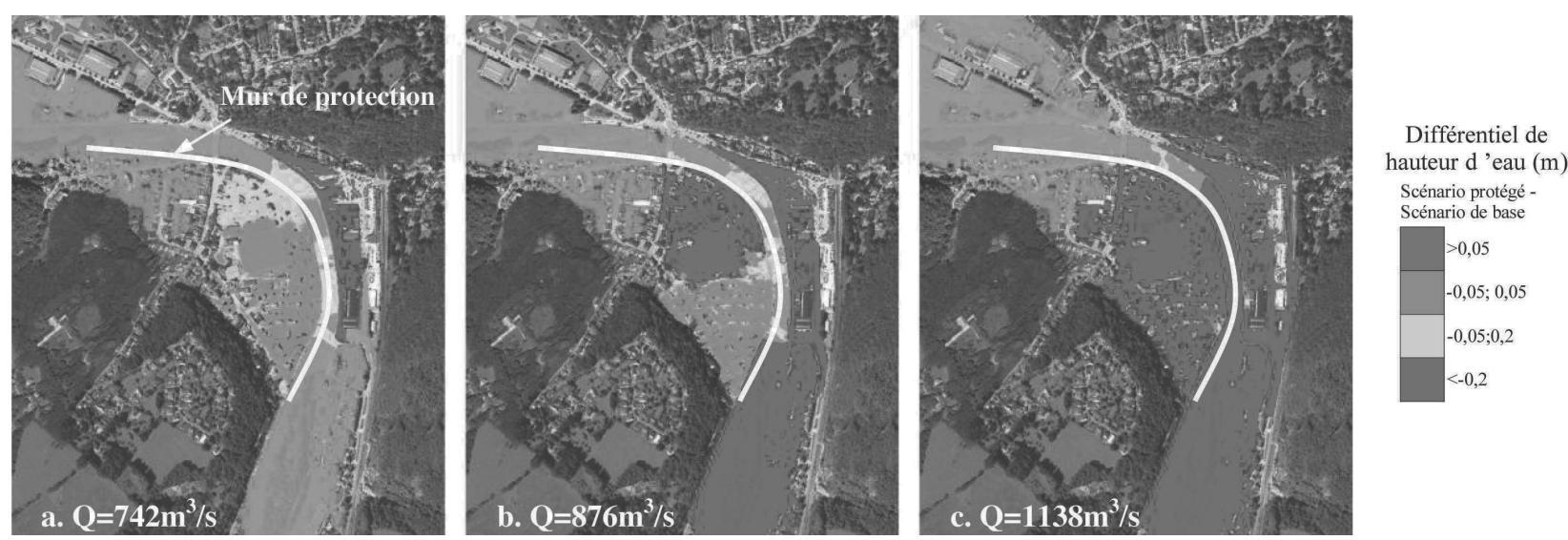

Figure 1 : Différentiel de hauteur d'eau entre la situation protégée et la situation actuelle pour le cas d'étude de la rehausse du mur de protection à Esneux pour trois débits représentatifs.

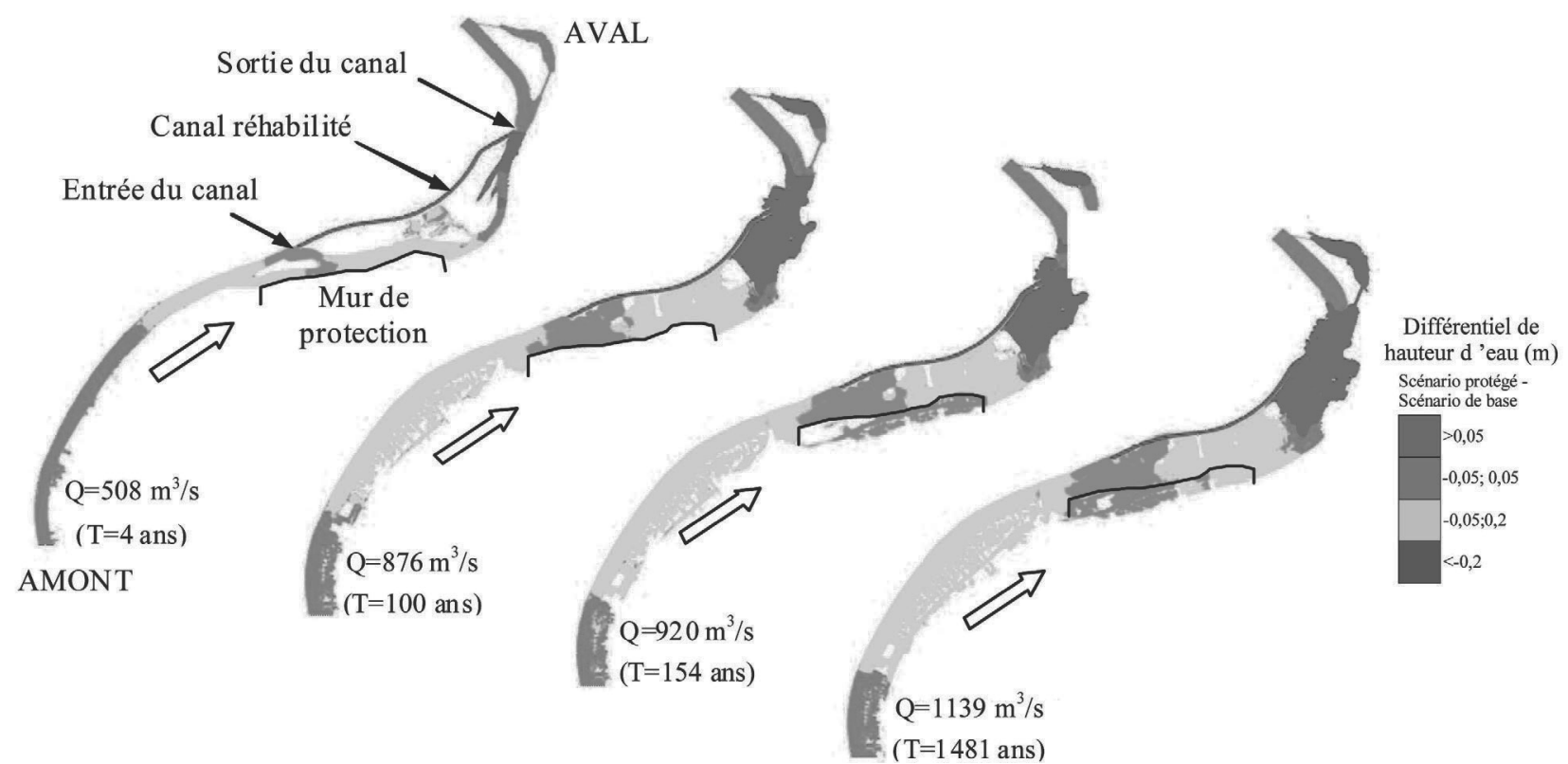

Figure 2 : Différentiel de hauteur d'eau entre la situation protégée et la situation actuelle pour le cas d'étude de la réhabilitation de l'ancien canal à Tilff pour quatre débits représentatifs.

sur l'attribut «adresse postale » de chacune des bases de données. Néanmoins, une visite de terrain reste indispensable afin de lever certaines ambiguités résiduelles à la suite de l'agrégation automatique des deux bases de données. Ces données ou d'autres sources d'information comparables sont disponibles partout en Belgique, ce qui garantit l'application de la méthodologie sur l'ensemble du territoire.

Suite à l'agrégation des deux sources d'information, l'objet soumis au risque est localisé et différentes propriétés lui sont assignées, dont les paramètres hydrauliques utiles pour la suite l'analyse et le type de bâtiment (résidentiel, commercial, public, industriel, ...). La Figure 3 montre l'identification individuelle des biens soumis au risque dans le village de Poulseur, dans le cadre de l'activation d'une plaine inondable passive.

\section{IV.5. Evaluation des impacts socio-économiques}

L'évaluation des impacts socio-économiques nécessite l'utilisation de sources de données complémentaires (Institut National des Statistiques, ...), qui permettent d'assigner de nouvelles propriétés identifiées lors de l'analyse de l'expo- sition (coût estimé du bien, nombre de personnes habitant le bien, vulnérabilité sociale, capacité d'adaptation, ...). Le coût estimé des bâtiments d'habitation $(P I)$ est évalué par un polynôme du second degré en fonction du revenu cadastral $(C I)$. Les coefficients ont été déterminés sur base de prix de vente de biens obtenus auprès d'agents immobiliers pour des habitations situées dans les trois villages étudiés : $P=160000-50 C I+0.125 C I^{2}$. Un résultat d'estimation du dommage économique est illustré à la Figure 5 dans le cas de la réhabilitation du canal.

Quant à la vulnérabilité sociale des personnes, elle a été estimée par Coninx et al. [8] en suivant la méthodologie synthétisée ci-avant. Les résultats d'impacts sociaux sont ici exposés selon deux représentations distinctes : soit de manière distribuée permettant l'identification de chaque bâtiment et la localisation spatiale de zones problématiques (Figure 3), soit sous la forme de courbes du nombre cumulé de personnes touchées en fonction de la probabilité de dépassement permettant la représentation des impacts pour toutes les récurrences de crue (Figure 4).

Les figures 1 à 4 illustrent l'étendue des résultats disponibles et permettent une analyse comparative des trois 

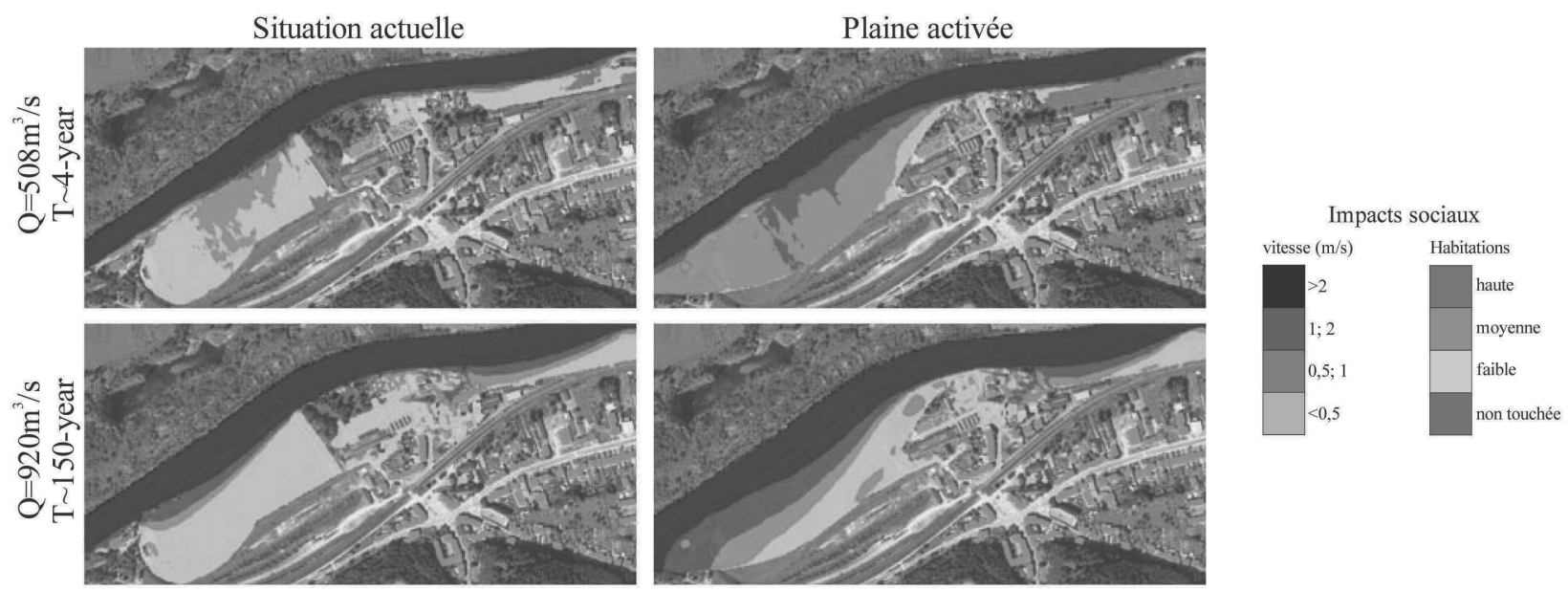

Figure 3 : Champ de vitesse et classe d'impact social des habitations à Poulseur pour deux débits représentatifs.
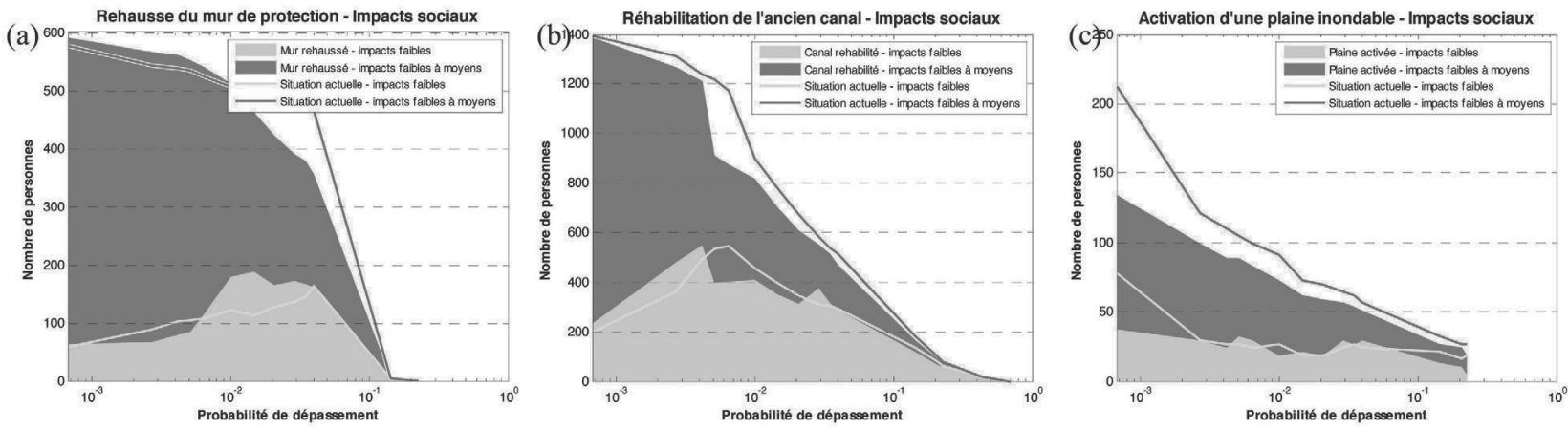

Figure 4 : Évolution des impacts sociaux pour les trois mesures considérées, nombre de personnes par classe de vulnérabilité sociale.

mesures étudiées. En ce qui concerne la rehausse du mur de protection (Figure 4a.), une inversion de l'effet de la mesure aux environs du débit centennal apparaît. L'influence de la mesure devient alors défavorable sur le nombre de personnes touchées dans chaque catégorie de vulnérabilité sociale. Ceci provient du fait que pour ce type de débits, une mise en charge se produit à l'amont du mur. La rehausse devient alors un obstacle supplémentaire à l'écoulement qui induit un déversement accru au dessus du mur dans sa partie amont (Figure 1). L'intérêt d'une approche de dimensionnement « orientée risque » est évident dans ce cas. En effet, si l'évaluation avait été effectuée sur une seule période de retour fixée entre la crue décennale et la centennale, la rehausse du mur de protection se serait avérée favorable. Par contre, si l'on considère l'ensemble des récurrences, le constat diverge.

L'étude de la réhabilitation de l'ancien canal (Figure 4b.) révèle une diminution du dommage de l'ordre de 10 à $15 \%$ pour toutes les récurrences, à l'exception des périodes de retour entre 100 et 200 ans, pour lesquelles la réduction atteint $25 \%$ du dommage initial (Figure 5c.). Grâce à l'augmentation de la section de l'Ourthe à cet endroit, les hauteurs d'eau au niveau du mur sont plus faibles pour un même débit dans cette gamme, tandis qu'elles augmentent à l'amont de la sortie du canal, où il n'y a pas d'enjeu (Figure 5).

Enfin, l'analyse des effets de l'activation de la plaine inondable passive sur la courbe de vulnérabilité sociale révèle une autre dynamique. En effet, le niveau de protection induit par la mesure progresse monotonement avec le débit de crue (Figure 3 et Figure 4c.). Plus le débit augmente, plus la mesure est effective. Encore une fois, seule une analyse basée sur l'examen d'une large gamme d'évènements hydrologiques permet de dégager ce type de tendance.

\section{IV.6. Analyse de sensibilité}

Outre les incertitudes associées à la modélisation de l'aléa inondation, un autre facteur important influençant l'incertitude globale de l'évaluation du risque réside dans l'évaluation de la valeur des paramètres hydrauliques assignés aux objets touchés par l'inondation, et particulier la hauteur d'eau. L'origine de cette incertitude provient du manque de données disponibles pour déterminer avec exactitude le niveau à partir duquel l'objet soumis au risque devient vulnérable à l'inondation.

Plusieurs méthodes d'assignation des paramètres hydrauliques ont été testées selon deux voies principales: soit les paramètres hydrauliques sont déterminés par une analyse statistique des mailles voisines de l'objet (Figure 5a.); soit, l'altitude de la surface du sol et celle de la surface libre sont interpolées selon un plan à l'intérieur du bâtiment (Figure 5b.) À ce stade des recherches, l'étude montre que l'interpolation selon un plan à au voisinage des objets, soit par une méthode des moindres carrés soit une méthode basée sur la détection des valeurs aberrantes (RANSAC), peut s'avérer prometteuse. En l'absence de données complémentaires, deux autres méthodes d'analyse du voisinage (moyenne de toutes mailles voisines et maximum absolu dans le voisinage de l'objet) ont été utilisées pour fournir, respectivement, une borne inférieure et une borne supérieure de la valeur 

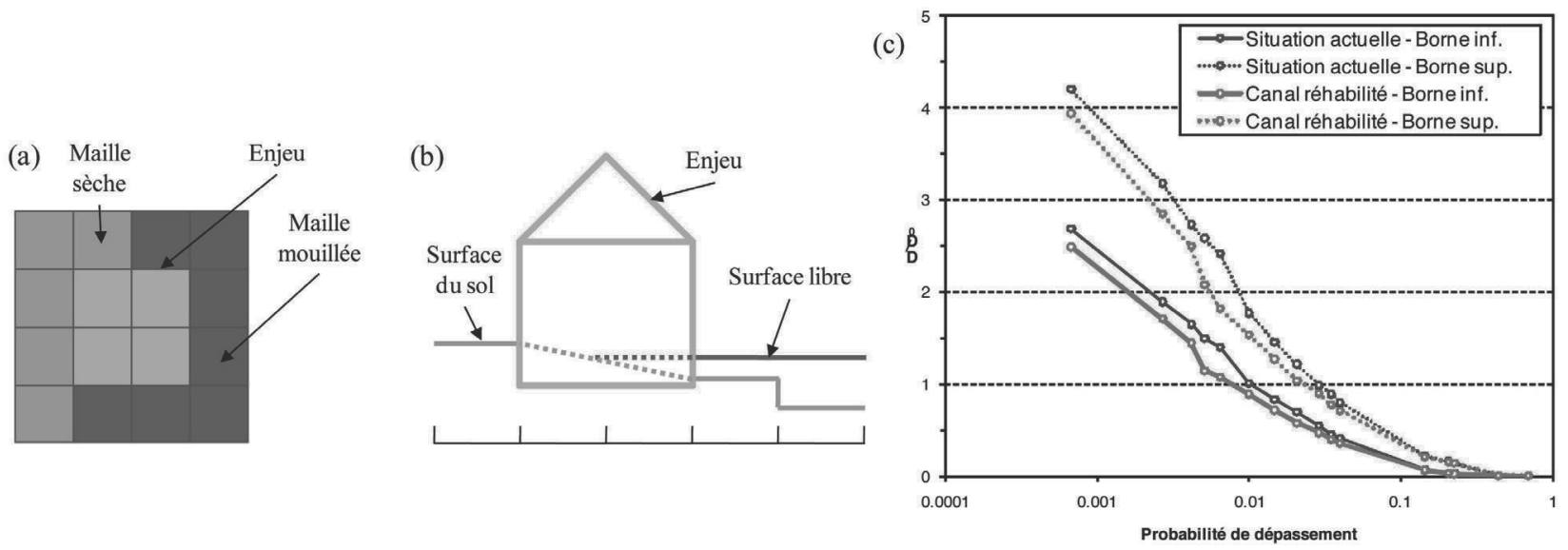

Figure 5 : Comparatif des méthodes d'assignation des paramètres hydrauliques aux objets soumis au risque (a. analyse du voisinage b. interpolation selon un plan). c. Évolution du rapport du dommage direct tangible pour chaque récurrence - D, par rapport au dommage évalué pour le crue centennale et le scenario de base $-D_{0}$.

de hauteur d'eau affectant les bâtiments. Dès lors, ces deux valeurs devront être considérées dans l'analyse de risque afin de rendre compte de l'incertitude sur la détermination du niveau à partir duquel l'enjeu est effectivement inondé.

Afin d'illustrer ce point, la Figure 5c. présente l'évolution du dommage économique pour la réhabilitation du canal. Le trait plein représente le cas où les hauteurs d'eau sont assignées par moyenne des hauteurs d'eau (borne inférieure) et le trait pointillé correspond à la recherche du maximum (borne supérieure). Le dommage tangible direct est exprimé sous la forme du rapport entre la valeur de dommage potentiel (D), et le dommage évalué pour la crue centennale dans le cas du scénario de base avec l'évaluation de la hauteur d'eau par la moyenne $\left(\mathrm{D}_{0}\right)$. Incontestablement, une différence marquée demeure entre les résultats fournis par les deux approches.

\section{CONCLUSIONS}

La communication illustre une méthodologie innovante d'évaluation du risque à micro-échelle combinée à une modélisation hydraulique bidimensionnelle. Elle met également en exergue les avantages d'une approche "orientée risque » pour le dimensionnement de tout ouvrage de protection contre les inondations et les manquements pouvant apparaître lorsque des méthodes basées sur une crue unique de dimensionnement sont utilisées. Les perspectives d'évolution de la méthodologie résident essentiellement dans la quantification des incertitudes sur l'ensemble de la filière (courbe hydrologique, modélisation hydraulique, modélisation de l'exposition et de la vulnérabilité socio-économique) et le report de celles-ci sur les courbes de risque.

\section{RÉFÉRENCES}

[1] Kaplan S. \& Garrick B. J. (1981) - On The Quantitative Definition of Risk. Risk Analysis. 1(1) 11-27

[2] Khuat Duy B., Archambeau P., Dewals B. J., Erpicum S. \& Pirotton M. - River modelling and flood mitigation in a Belgian catchment. Proc. Inst. Civil. Eng.-Water Manag. 163(8) $417-423$

[3] Apel H., Aronica G., Kreibich H. \& Thieken A. (2009) Flood risk analyses - how detailed do we need to be ? Natural Hazards. 49(1) 79-98
[4] Mcmillan H. K. \& Brasington J. (2008) — End-to-end risk assessment : A coupled model cascade with uncertainty estimation. Water Resources Research, 44(W03419) 14

[5] Ernst J., Dewals B. J., Detrembleur S., Archambeau P., Erpicum S. \& Pirotton M. (2010) - Micro-scale flood risk analysis based on detailed 2D hydraulic modelling and high resolution geographic data. Nat. Hazards. 55(2) 181-209

[6] ConinX I. \& Bachus K. (2007) - Integrating social vulnerability to floods in a climate change context. Proc. Int. Conf. on adaptive and integrated water management, coping with complexity and uncertainty. Basel, Switzerland

[7] Ernst J., Coninx I., Dewals B. J., Detrembleur S., Erpicum S., Pirotton M. \& Bachus K. (2009) - Planning flood risk reducing measures based on combined hydraulic simulations and socio-economic modelling at a micro-scale. Proc. European Water Ressources Association 7th Int. Conf. - Water Resources Conservation and Risk Reduction Under Climatic Instability. Limassol, Cyprus

[8] Ernst J., Coninx I., Dewals B. J., Detrembleur S., Erpicum S., Bachus K. \& Pirotton M. (2009) - Social Flood Impacts in Urban Areas : Integration of Detailed Flow Modelling and Social Analysis. Proc. 33rd IAHR Congress - Water engineering for a sustainable environment. 2009, IAHR: Vancouver, British Columbia

[9] Sмітн D. I. (1994) - Flood damage estimation - A review of urban stage damage curves and loss function. Water SA. 20(3) 231-238

[10] Icpr (2008) - Le Rhin : Un fleuve renoue contact : ICPR $32 \mathrm{p}$.

[11] Dushmanta D., SRikantha H. \& Katumi M. (2003) - A mathematical model for flood loss estimation. J. Hydrol. 277 24-49

[12] Penning-Rowsell E. C., Johnson C., Tunstall S. M., Tapsell S. M., Morris J., Chatterton J. B., Coker A. \& Green C. (2003) - The Benefits of Flood and Coastal Defence : Techniques and Data for 2003. Flood Hazard Research Centre, Middlesex University

[13] Büchele B., Kreibich H., Kron A., Thieken A., Ihringer J., Oberle P., Merz B. \& Nestmann F. (2006) - Floodrisk mapping: contributions towards an enhanced assessment of extreme events and associated risks. Natural Hazards and Earth System Science. 6(4) 485-503

[14] Kreibich H., Thieken A. H., Petrow T., Müller M. \& Merz B. (2005) - Flood loss reduction of private households due to building precautionary measures? lessons learned from the Elbe flood in August 2002. Natural Hazards and Earth System Science. 5(1) 117-126 
[15] Thieken A. H., Müller M., Kreibich H. \& Merz B. (2005) - Flood damage and influencing factors: New insights from the August 2002 flood in Germany. Water Resour. Res. 41(W12430)

[16] De Wit M. J. M., Peeters H. A., Gastaud P. H., Dewil P., Maeghe K. \& Baumgart J. (2007) - Floods in the Meuse basin : event descriptions and an international view on ongoing measures. Int. J. River Basin Mang. 5(4) 279-292

[17] Dewals B. J., Erpicum S., Archambeau P., Detrembleur S. \& Pirotton M. (2006) — Depth-integrated flow modelling taking into account bottom curvature. J. Hydraul. Res. 44(6) 787-795

[18] Roger S., Dewals B. J., Erpicum S., Schwanenberg D., Schüttrumpf H., Köngeter J. \& Pirotton M. (2009) -
Experimental und numerical investigations of dike-break induced flows. J. Hydraul. Res. 47(3) 349-359

[19] Dewals B. J., Kantoush S. A., Erpicum S., Pirotton M. \& Schleiss A. J. (2008) - Experimental and numerical analysis of flow instabilities in rectangular shallow basins. Environ Fluid Mech. 8 31-54

[20] Erpicum S., Dewals B. J., Archambeau P., Detrembleur S. \& Pirotton M. (2010) - Detailed inundation modelling using high resolution DEMs. Engineering Applications of Computational. Fluid Mechanics. 2(4) 196-208

[21] Erpicum S., Meile T., Dewals B. J., Pirotton M. \& Schleiss A. J. (2009) - 2D numerical flow modeling in a macro-rough channel. Int. J. Numer. Methods Fluids. 61(11) 1227-1246 